\title{
Modified transventricular and transaortic mitral valve edge-to-edge repair mimicking MitraClip overcorrection
}

Nestoras Papadopoulos, MD, PhD, ${ }^{\mathrm{a}}$ Omer Dzemali, MD, PhD, ${ }^{\mathrm{a}}$ Luisa Bott, $\mathrm{MD},{ }^{\mathrm{b}}$

Vasileios Ntinopoulos, $\mathrm{MD},{ }^{\mathrm{a}}$ Aleksandra Miskovic, $\mathrm{MD},{ }^{\mathrm{b}}$ and Anton Moritz, $\mathrm{MD}, \mathrm{PhD}^{\mathrm{c}}$

\section{ABSTRACT}

Objective: In the current study, we present our mid-term experience with modified edge-to-edge repair technique through a transventricular and transaortic route in patients requiring left ventricular remodeling or aortic root/valve surgery.

Methods: From December 2006 through April 2015, 49 high-risk patients (median age: 69 years; median European System for Cardiac Operative Risk Evaluation II: 11.4 [6.54-14.9]) underwent transventricular $(N=7 ; 14 \%)$ or transaortic $(N=42 ; 86 \%)$ edge-to-edge mitral valve repair. The Alfieri stitch technique was modified by MitraClip type overcorrection and solid buttressing behind the posterior leaflet. Indication was grade $2+$ functional mitral valve incompetence and dilated or impaired left ventricle $(\mathrm{N}=25 ; 52 \%)$, or grade $3+(\mathrm{N}=22 ; 45 \%)$ and grade $4+$ functional mitral valve regurgitation $(\mathrm{N}=2 ; 4 \%)$. Surgical procedure included aortic root surgery in $65 \%$, aortic valve replacement with surgical revascularization in $18 \%$, and Dorplasty with surgical revascularization in $14 \%$.

Results: Intraoperative mortality and early neurologic complications were absent in our series. Ninety-day mortality was $12.2 \%(\mathrm{~N}=6)$. Median clinical and echocardiographic follow-up-time was 50.7 (21.5-44.1) and 39.2 (33.7-44.1) months, respectively. Median postoperative transvalvular gradient was low $(2.72$ [1.91-4.22] mm Hg) and did not increase during follow-up $(P=.268)$, although peak gradient rose slightly from 7.41 to $8.12 \mathrm{~mm} \mathrm{Hg}(P=.071)$. The actuarial reoperation free rate at the index valve was $96.8 \%$.

Conclusions: Transventricular or transaortic Alfieri mitral repair mimicking mitral clip overcorrection represents a quick and safe technique in the setting of high-risk patients undergoing left ventricular remodeling or aortic root/valve surgery and can be performed with low risk of creating mitral stenosis at midterm. The technique is straightforward, with reliable identification of the center of the valve leaflets being the limitation. (JTCVS Techniques 2022;12:39-51)

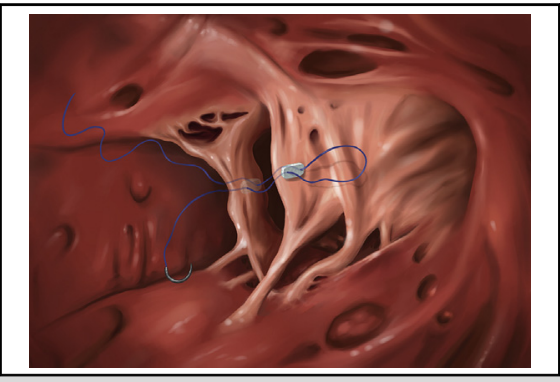

Modified mitral valve edge-to-edge repair mimicking MitraClip overcorrection.

CENTRAL MESSAGE

Modified transventricular or

transaortic Alfieri mitral repair

represents a straightforward

technique that can be performed

with low risk of creating mitral

stenosis at midterm.

\section{PERSPECTIVE}

Transventricular or transaortic Alfieri mitral repair is a safe and straightforward technique. Reliable identification of the center of the valve leaflets represents a limitation. The effectiveness of the technique may be improved by MitraClip type overcorrection and solid buttressing behind the posterior leaflet.

See Commentary on page 52.

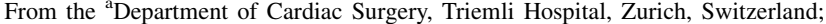
${ }^{\mathrm{b}}$ Department of Thoracic and Cardiovascular Surgery, Goethe University Frankfurt/Main, Germany; and ${ }^{c}$ Department of Thoracic and Cardiovascular Surgery, Kepler University Hospital, Linz, Austria.

Drs Papadopoulos and Dzemali contributed equally to this study.

Received for publication Feb 16, 2021; accepted for publication Jan 12, 2022; available ahead of print Jan 19, 2022.

Address for reprints: Nestoras Papadopoulos, MD, PhD, Department of Cardiac Surgery, Triemli Hospital, Birmensdorferstrasse 497, 8063 Zurich, Switzerland (E-mail: nestoras.papadopoulos@triemli.zuerich.ch).

2666-2507

Copyright $@ 2022$ The Author(s). Published by Elsevier Inc. on behalf of The American Association for Thoracic Surgery. This is an open access article under the CC BY-NC-ND license (http://creativecommons.org/licenses/by-nc-nd/4.0/).

https://doi.org/10.1016/j.xjtc.2022.01.005
- Video clip is available online.

Mitral valve repair represents the gold standard for restoration of normal valve function in a setting of severe primary nonrheumatic mitral valve regurgitation. ${ }^{1-10}$ Recommendations regarding the concomitant treatment of moderate or moderate-to-severe secondary mitral valve regurgitation in patients requiring left-sided heart surgery 


\section{Abbreviations and Acronyms \\ $\mathrm{CABG}=$ coronary artery bypass grafting \\ CI $=$ confidence interval \\ EUROScore $=$ European System for Cardiac \\ Operative Risk Evaluation \\ NYHA $=$ New York Heart Association}

are vaguely defined in the current guidelines for the management of valvular heart disease. ${ }^{1,2}$

Moderate or moderate-to-severe secondary mitral valve regurgitation may present as a concomitant finding in patients requiring a challenging aortic root/valve or left ventricular remodeling procedure. ${ }^{11-14}$ This particular patient cohort is usually characterized by high-risk profile, and the related procedures are mostly time-consuming. ${ }^{15}$ As postoperative persistence of mitral valve regurgitation has been shown to affect survival adversely, a concomitant mitral valve approach may be added to a challenging aortic root/valve or left ventricular remodeling procedure to avoid that risk. ${ }^{16-18}$ In these cases, reduction of surgical time spent for mitral repair is key.

Fucci and associates ${ }^{19}$ presented a simple and timesaving edge-to-edge repair technique through single suture approximation of anterior and posterior leaflet as a reproducible and effective tool for restoration of mitral valve competence in 1995. In 2006, we started using the edgeto-edge repair through a transventricular or transaortic route in high-risk patients requiring left ventricular remodeling or complex aortic root/valve surgery.

Maisano and associates ${ }^{20}$ reported suboptimal midterm results following edge-to-edge repair technique when annuloplasty ring is not added to the repair. As annuloplasty is neither through the transaortic nor through the transventricular access feasible, we modified the Alfieri stitch technique by mimicking a MitraClip style overcorrection that may lead to a reduction in the anteroposterior diameter of the mitral annulus. We hypothesized that modified Alfieri stich technique would be associated with low rate of severe mitral valve stenosis at mid-term follow-up. The current study highlights the medium-term results of this procedure with clinical and echocardiographic follow-up data in a cohort consisting of 49 high-risk patients.

\section{METHODS \\ Patient Selection}

Patients requiring aortic root surgery or left ventricular remodeling due to aortic root or left ventricular aneurysm in the presence of a moderate or moderate-to-severe secondary mitral valve regurgitation were eligible for transaortic or transventricular edge-to-edge repair. The need to make an extra left atrial incision, the commonly laterally displacement of the mitral annulus due to aortic root/left ventricular dilatation, and frequently small left atrium in these cases make the left atrial route unfavorable. In such scenario, edge-to-edge repair may represent a quick and safe alternative to left atrial approach, which according to the actuarial literature neither prolongs the crossclamp time significantly nor adds to the technical complexity of the procedure. ${ }^{21-23}$ Figure E1 illustrates a Consolidated Standards of Reporting Trials diagram of our study. Inclusion criteria consisted of highrisk profile with aortic root or left ventricular aneurysm with concomitant grade $2+$ functional mitral valve incompetence and dilated or impaired left ventricle or concomitant grade $3+$ and grade $4+$ functional mitral valve regurgitation. Patients with primary mitral valve incompetence and with low- or intermediate-risk profile were excluded from the study. For the calculation of the predicted operative mortality in our series, the European System for Cardiac Operative Risk Evaluation (EUROScore) II was used. ${ }^{24}$

The primary end point of the current study was the incidence of severe mitral valve stenosis at midterm follow-up. Secondary end points included beside perioperative and midterm morbidity and mortality, the distribution of the New York Heart Association (NYHA) class, and the incidence of moderate and severe mitral valve regurgitation following edge-to-edge repair through a transaortic and transventricular route. The local ethics committee approved the study protocol. (No. 79/13; approval date: October $15,2018)$, and individual patient consent was obtained. Patients provided informed written consent for the publication of their study data.

\section{Operative Technique}

Our operative technique included a standard ascending aortic- and bicaval venous cannulation. A left ventricular vent was inserted via the right superior pulmonary vein. Cardiopulmonary bypass was performed in moderate hypothermia $\left(32^{\circ} \mathrm{C}\right)$, and cardiac arrest was achieved using antegrade and retrograde tepid blood cardioplegia.

\section{Transventricular Approach}

A left ventricular incision was made preferably in the center of scarred ventricular tissue and as far as possible away from relevant coronary arteries. Access to the mitral valve was obtained after placing stay sutures at the left ventricular wall. Figure 1 illustrates the transventricular view to the mitral valve where A2 and P2 segment of the anterior and posterior mitral leaflet can be identified. They are located at the watershed of chordae tendinae originating from anterolateral and posteromedial papillary muscle, respectively, as demonstrated in Figure 1. A pledget reinforced 4/0 Cardionyl $\mathrm{U}$ suture was passed close to the annulus of the P2 segment and then passed through a corresponding point of the anterior leaflet but closer to the free edge. The second arm of the suture was then passed closer to the free margin of both leaflets and tied. The free edge adaptation was secured by 2 more over and over stitches. Finally, ventricular restoration was performed using a Dor-plasty.

\section{Transaortic Approach}

Following crossclamping of the ascending aorta, exposition of the aortic valve was achieved through a transverse aortotomy. If necessary, resection of the aortic sinuses with mobilization of the coronary ostia as buttons was performed, to prepare the situs for aortic root replacement with reimplantation or replacement of the aortic valve. Following the aortomitral curtain, the primary chordae tendineae of the anterior mitral leaflet at A2 segment were pulled laterally and apically (Figure 2), hereby the zenith of the watershed created from the primary chordae tendineae originating from the anterolateral and posteromedial pupillary muscle reaching the posterior mitral leaflet can usually be identified, indicating P2 segment, as illustrated in Figure 2. In rare cases with a fan-shaped flat posterior papillary muscle (Figure E2), the center of the posterior leaflet may by hard to identify. In these cases, the center is usually the highest point of the segment, as 


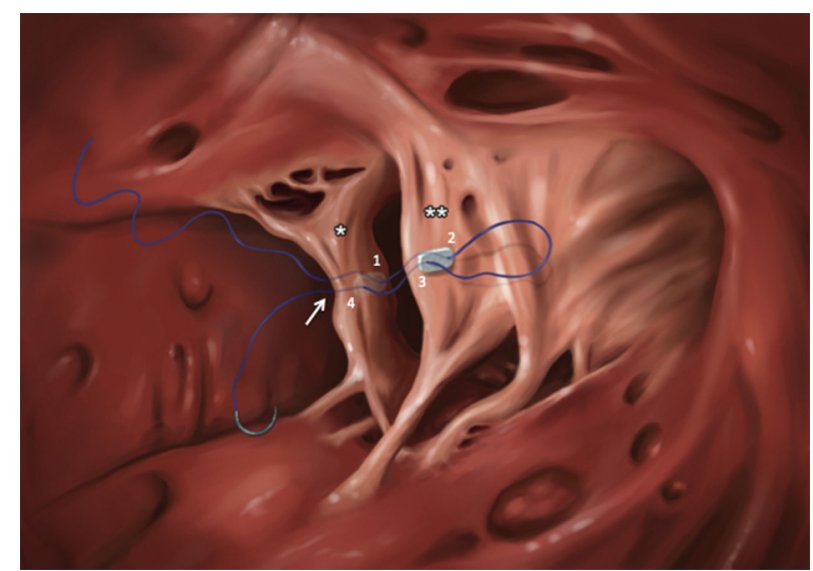

FIGURE 1. Transventricular surgical view of the mitral valve showing the posterior (single white star) and anterior mitral leaflet (double white star). A left ventricular incision was made preferably in the center of scarred ventricular tissue and as far as possible away from relevant coronary arteries. Access to the mitral valve was obtained after placing stay sutures at the left ventricular wall (not shown). The P2 segment (white arrow) is located at the watershed of chordae tendinae, originating from anterolateral and posteromedial papillary muscle. A pledget-reinforced 4/0 Cardionyl U-suture was passed close to the annulus of the $\mathrm{P} 2$ segment (position 1) and then passed through a corresponding point of the anterior leaflet but closer to the free edge (position 2). The second arm of the suture was then passed closer to the free margin of both leaflets and tied (position 3 and 4). In this manner, effectivity of the technique can be improved by mimicking a MitraClip style overcorrection through solid buttressing behind the posterior leaflet that may lead to a reduction in the anteroposterior diameter of the mitral annulus.

illustrated in Figure E2. Subsequently, stitches are placed as described previously.

\section{Follow-up}

All survivors were followed in our outpatient clinic. Detailed information regarding functional status, NYHA class, valve-related complications, and present medication were recorded. Additional data were acquired from referring physicians.

\section{Statistical Analysis and Data Collection}

The statistical analyses were performed with SPSS, version 25 (IBM Corp, Armonk, NY) and R version 3.6.3 (R Foundation for Statistical Computing, Vienna, Austria). Categorical variables are expressed as counts and percentages and were evaluated with the $\chi^{2}$ or the Fisher exact test. Assessment of distribution normality was performed mainly graphically with the use of histograms, probability-probability plots and quantilequantile plots and supplementary with the use of Shapiro-Wilk and Kolmogorov-Smirnov tests. Data of continuous variables were compared with the $t$ test for normally distributed data or the Mann-Whitney $U$ test for non-normally distributed data. Skewed distributions presented as median (quartile 1, quartile 3). Longitudinal analysis of serial echocardiographic data was performed with a mixed-effects ordinal logistic regression model to estimate the proportion of patients in each mitral valve stenosis grade over time. The longitudinal analysis was performed using $\mathrm{R}$ with the GLMM adaptive package. Cases with missing data were handled with pairwise deletion.

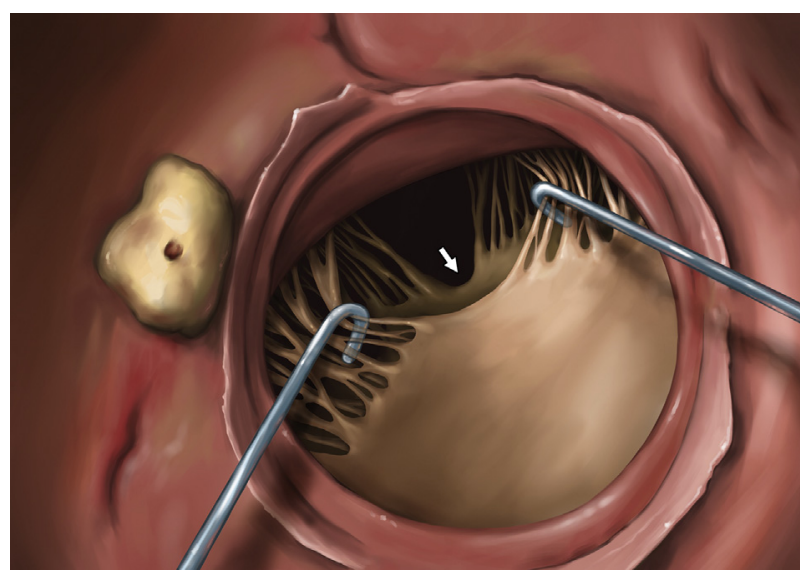

FIGURE 2. Transaortic surgical view of the anterior mitral leaflet, following preparation of the aortic root for the Bentall's procedure. The button of the right coronary artery can be seen on the left side. Following the aortomitral curtain, the primary chordae tendineae of the anterior mitral leaflet at A2 segment were pulled laterally and apically through nerve hooks as shown, hereby the zenith of the watershed (white arrow) created from the primary chordae tendineae originating from the anterolateral and posteromedial pupillary muscle reaching the posterior mitral leaflet can easily be identified, indicating the P2 segment. Subsequently, stitches were placed as described in Figure 1. In summary, a pledget-reinforced 4/0 Cardionyl U-suture was passed close to the annulus of the P2 segment and then passed through a corresponding point of the anterior leaflet but closer to the free edge. The second arm of the suture was then passed closer to the free margin of both leaflets and tied.

\section{RESULTS}

\section{Demographic Data}

Baseline characteristics of our patient cohort are summarized in Table 1. Preoperative echocardiographic data are illustrated in Table 2. Twenty-five patients had grade $2+$ $(\mathrm{N}=25,52 \%), 22$ patients grade $3+(45 \%)$, and 2 patients $(4 \%)$ had grade $4+$ mitral valve regurgitation. Preoperative left ventricular ejection fraction was $45.2 \pm 14.2 \%$ and median left ventricular end-diastolic diameter $57.5 \mathrm{~mm}$ (49.065.2). Further preoperative echocardiographic findings included severe aortic valve regurgitation in 26 patients $(53 \%)$, severe aortic valve stenosis in 9 patients $(18.4 \%)$, and severe tricuspid valve regurgitation in 6 patients $(12 \%)$. All patients underwent coronary angiography preoperatively, and 27 patients $(55 \%)$ showed coronary artery disease of varying severity. Coronary 3-vessel disease was detected in 13 patients $(26.5 \%)$, whereas coronary 2 - and 1 -vessel disease were found in $6(12 \%)$ and 8 patients $(16 \%)$, respectively. Echocardiographic examination as well as magnetic resonance tomography before surgery revealed a left ventricular aneurysm in 7 patients $(14 \%)$.

From December 2006 through April 2015, a total of 49 patients with a mean age of $68 \pm 11$ years underwent concomitant modified edge-to-edge mitral valve repair through a transventricular $(\mathrm{N}=7 ; 14 \%)$ or a transaortic 
TABLE 1. Baseline characteristics

\begin{tabular}{|c|c|c|}
\hline \multirow[b]{2}{*}{ Variables } & \multicolumn{2}{|c|}{ Total patient cohort $(n=49)$} \\
\hline & No. & $\%$ \\
\hline Age, y & \multicolumn{2}{|c|}{$68 \pm 11$} \\
\hline Female & 15 & 31 \\
\hline Median EUROScore II, n (\%) & \multicolumn{2}{|c|}{$11.4(6.54-14.9)$} \\
\hline Atrial fibrillation & 18 & 37 \\
\hline Diabetes & 15 & 31 \\
\hline Arterial hypertension & 36 & 73.5 \\
\hline Pulmonary hypertension & 34 & 69 \\
\hline Previous stroke & 6 & 12 \\
\hline Coronary artery disease & 30 & 61 \\
\hline Peripheral vascular disease & 6 & 12 \\
\hline Cerebrovascular disease & 5 & 10 \\
\hline COPD & 11 & 22 \\
\hline Chronic renal failure & 18 & 37 \\
\hline Permanent pacemaker or ICD & 5 & 10 \\
\hline NYHA classification I-II & 2 & 4 \\
\hline NYHA classification III-IV & 47 & 96 \\
\hline
\end{tabular}

Chronic renal failure $=$ glomerular filtration rate $<60 \mathrm{~mL} / \mathrm{min} / 1.73 \mathrm{~m}^{2}$. EUROScore II, European System for Cardiac Operative Risk Evaluation II; COPD, chronic obstructive pulmonary disease; ICD, implantable Cardioverter Defibrillator; NYHA, New York Heart Association.

$(\mathrm{N}=42 ; 86 \%)$ route. Etiology of mitral valve regurgitation included valvular or ischemic cardiomyopathy leading to a secondary mitral valve insufficiency $(\mathrm{N}=49 ; 100 \%)$. Median EUROScore II of our patient cohort counted $11.4 \%$ $(6.54 \%-14.9 \%)$, which corresponds to a high-risk patient collective.

TABLE 2. Preoperative and follow-up echocardiographic data

\begin{tabular}{|c|c|c|c|c|}
\hline \multirow{2}{*}{ Variables } & Preoperative & \multicolumn{2}{|c|}{ At latest follow-up } & \multirow[b]{2}{*}{$P$ value } \\
\hline & \multicolumn{3}{|c|}{ Median (interquartile range) } & \\
\hline LVEF, \% & $45.0(32.1-57.4)$ & \multicolumn{2}{|c|}{$51.1(40.1-62.1)$} & .036 \\
\hline LVEDD, mm & $57.5(49.0-65.2)$ & \multicolumn{2}{|c|}{$52.1(47.3-59.2)$} & .074 \\
\hline LVESD, mm & $43.0(32.6-50.1)$ & \multicolumn{2}{|c|}{$37.5(26.1-43.5)$} & 089 \\
\hline Max MVP, mm Hg & $7.41(4.12-9.72)$ & \multicolumn{2}{|c|}{$8.12(6.21-13.7)$} & .071 \\
\hline Mean MVP, mm Hg & $2.72(1.91-4.22)$ & \multicolumn{2}{|c|}{$3.02(2.12-4.51)$} & .268 \\
\hline Mean MVA, $\mathrm{cm}^{2}$ & $2.43(1.72-2.91)$ & \multicolumn{2}{|c|}{$2.22(2.04-2.92)$} & .843 \\
\hline $\begin{array}{l}\text { Left atrial } \\
\quad \text { diameter, mm }\end{array}$ & $44.4(35.4-51.7)$ & \multicolumn{2}{|c|}{$45.2(39.2-51.5)$} & .804 \\
\hline \multicolumn{5}{|c|}{ Mitral valve regurgitation grade } \\
\hline None & 0 & 3 & $9.3 \%$ & \\
\hline $1+$ & 0 & 18 & $56.2 \%$ & \\
\hline $2+$ & $52 \%$ & 8 & $25.0 \%$ & \\
\hline $3+$ & $45 \%$ & 3 & $9.3 \%$ & \\
\hline $4+$ & $4 \%$ & 0 & 0 & \\
\hline
\end{tabular}

$L V E F$, Left ventricular ejection fraction; $L V E D D$, left ventricular end diastolic diameter; $L V E S D$, left ventricular end systolic diameter; $M V P$, mitral valve pressure gradient; $M V A$, mitral valve area.
Almost one-half of the procedures $(\mathrm{N}=20 ; 40.8 \%)$ were performed urgently, and the remaining $59.2 \%(\mathrm{~N}=29)$ were performed in an elective setting. The majority of the procedures were performed through median sternotomy $(\mathrm{N}=37 ; 75.5 \%)$, whereas $24.4 \%$ of them were carried out via partial upper sternotomy $(\mathrm{N}=12)$.

\section{Overview of Surgical Procedures}

Coronary artery bypass grafting (CABG; mean graft number: 2.44) was performed in 27 cases $(55 \%)$, with 7 of them undergoing concomitant ventricular restoration (Dor-plasty $=14 \%)$. Thirty-two patients $(65 \%)$ underwent aortic root replacement with reimplantation (Tirone David's procedure, $\mathrm{N}=6$ ) or replacement (Bentall's procedure, $\mathrm{N}=26$ ) of the aortic valve. Furthermore, 9 patients $(18 \%)$ required aortic valve replacement with concomitant CABG. Six patients $(12 \%)$ required a tricuspid valve reconstruction. Occlusion of left atrial appendage was performed in the majority of the cases $(\mathrm{N}=39,79.6 \%)$. In 1 case $(2 \%)$, transaortic edge-to-edge repair was performed in a second pump run following replacement of the ascending aorta and $\mathrm{CABG}$ due to a progression of mitral valve regurgitation from moderate to severe. Figure E3 summarizes all surgical procedures.

\section{Perioperative Mortality and Morbidity}

There was no intraoperative mortality in our series. One procedure $(2 \%)$ had to be abandoned intraoperatively due to a high transvalvular gradient. This patient was successfully treated by takedown of the edge-to-edge approximation and performance of mitral valve reconstruction using a standard annuloplasty-ring during a second pump run. Thus, procedural success counted $98 \%$. Mean crossclamp time was $102 \pm 36.5$ minutes, and mean cardiopulmonary bypass time was $163 \pm 51$ minutes. One patient $(2 \%)$ required extracorporeal life support due to low cardiac output. Ninety-day mortality was $12.2 \%(\mathrm{~N}=6)$. Three patients died due to intestinal ischemia leading to multiorgan failure, and 1 patient with obesity died due to hemorrhagic shock after dislocation of the arterial cannula of extracorporeal life support placed in the femoral artery. Two further patients died of septic shock with consecutive multiorgan failure. There were no perioperative neurologic complications in our series. Furthermore, postoperative complications consisted of re-exploration for bleeding in $14 \%$ $(\mathrm{N}=7)$, gastrointestinal bleeding in $10 \%(\mathrm{~N}=5)$ and myocardial infarction in $2 \%(\mathrm{~N}=1)$ of the patients. There were no perioperative deep sternal or superficial wound infections. Table 3 summarizes perioperative data and outcomes of our patient cohort.

\section{Midterm Clinical Outcomes}

Median clinical follow-up time was 50.7 (21.5-44.1) months. Follow-up was $96 \%$ complete. During follow-up, 
TABLE 3. Perioperative data and outcomes

\begin{tabular}{|c|c|c|}
\hline \multirow[b]{2}{*}{ Variables } & \multicolumn{2}{|c|}{ Total patient cohort $(n=49)$} \\
\hline & No. & $\%$ \\
\hline \multicolumn{3}{|l|}{ Primary aortic indication $(n=42)$} \\
\hline Aneurysm & 32 & 76.1 \\
\hline Aortic insufficiency & 25 & 59.5 \\
\hline Aortic stenosis & 9 & 21.3 \\
\hline Mixed & 8 & 19.1 \\
\hline \multicolumn{3}{|c|}{ Primary left ventricular indication $(\mathrm{n}=7)$} \\
\hline Anterior aneurysm & 4 & 57.1 \\
\hline Lateral aneurysm & 3 & 42.8 \\
\hline \multicolumn{3}{|l|}{ Mitral regurgitation cause } \\
\hline Secondary & 49 & 100 \\
\hline \multicolumn{3}{|l|}{ Operation status } \\
\hline Urgent & 20 & 40.8 \\
\hline Elective & 29 & 59.2 \\
\hline \multicolumn{3}{|l|}{ Surgical access way } \\
\hline Partial upper sternotomy & 12 & 24.4 \\
\hline T. David's procedure & 6 & 50.0 \\
\hline Bentall's procedure & 6 & 50.0 \\
\hline Median sternotomy & 37 & 75.5 \\
\hline \multicolumn{3}{|l|}{ Concomitant procedures } \\
\hline Edge-to-edge repair & 49 & 100 \\
\hline CABG & 27 & 55.1 \\
\hline Tricuspid valve reconstruction & 6 & 12.2 \\
\hline Left atrial appendage occlusion & 39 & 79.5 \\
\hline Maze & 4 & 8.16 \\
\hline \multicolumn{3}{|l|}{ Outcome } \\
\hline Crossclamp time, $\min$ & \multicolumn{2}{|c|}{$102 \pm 36.5$} \\
\hline CPB time, $\min$ & \multicolumn{2}{|c|}{$163 \pm 51.0$} \\
\hline Chest tube drainage $24 \mathrm{~h}, \mathrm{~mL}$ & \multicolumn{2}{|c|}{$550 \pm 524$} \\
\hline Extracorporeal life support & 1 & 2.04 \\
\hline Reoperative bleeding & 7 & 14.2 \\
\hline Stroke permanent/transient & 0 & 0 \\
\hline Myocardial infarction & 1 & 2.04 \\
\hline Gastrointestinal bleeding & 5 & 10.2 \\
\hline Renal failure & 15 & 30.6 \\
\hline Deep sternal infection & 0 & 0 \\
\hline Ninety-day mortality & 6 & 12.2 \\
\hline
\end{tabular}

$C A B G$, Coronary artery bypass grafting; $C P B$, cardiopulmonary bypass.

$13(26.5 \%)$ patients of our cohort died. Causes of late mortality were septic shock following bilateral pneumonia or ileus $(\mathrm{N}=2)$, electrolyte derangement following dialysis $(\mathrm{N}=1)$, and multiorgan failure $(\mathrm{N}=2)$. Cardiac-related midterm mortality was detected in 2 patients, who died following myocardial infarction and heart failure. In 6 patients, the precise cause of death could not be detected. Based on Kaplan-Meier analysis (Figure E4), overall survival was $66.5 \pm 2 \%$ at 4 years. As highlighted in Figure E5, at follow-up 10 of the 30 survivors (33\%) were in NYHA functional class I, $16(54 \%)$ in class II, 3 $(10 \%)$ in class III, and 1 patient $(3 \%)$ in class IV. There was only $1(3 \%)$ major neurologic event (bleeding) during follow-up.

\section{Valve-Related Complications and Echocardiographic Follow-up}

One patient required reoperation on the index valve 1 year postoperatively due to tear out of the Alfieri stitch on the posterior leaflet. The primary procedure included aortic root replacement with biological conduit and concomitant mitral valve edge-to-edge repair. Reinforcement of the Alfieri stitch was performed with an autologous pericardial strip due to the presence of aortic valve endocarditis. The patient underwent mitral valve replacement 1 year postoperatively. Thus, actuarial reoperation free rate at the index valve was $96.8 \%$.

Median echocardiographic follow-up time was 39.2 (33.7-44.1) months and 96\% complete. At latest followup, 3 patients $(9 \%)$ showed no mitral valve regurgitation, 18 patients $(56 \%)$ had mitral valve regurgitation grade $1+, 8(25 \%)$ mitral valve regurgitation grade $2+$, and 3 patients $(9 \%)$ mitral valve regurgitation grade $3+$. No patient showed mitral valve regurgitation grade $4+$. Mean mitral valve orifice area at follow-up was $2.6 \pm 0.9 \mathrm{~cm}^{2}$. As summarized in Table 2, median postoperative transvalvular gradient was low (2.72 [1.91-4.22] mm Hg) and did not increase during follow-up $(P=.268)$ although peak gradient rose slightly from 7.41 to $8.12 \mathrm{~mm} \mathrm{Hg}(P=.071)$. Table E1 shows echocardiographic outcomes between transaortic and transventricular approach at latest Follow-up. The proportion of patients in each mitral valve stenosis grade over time is presented in Table 4 and illustrated in Figure 3. Four years postoperatively, none of the patients with echocardiographic follow-up had severe mitral valve stenosis, whereas $11.9 \%$ of the patients had moderate $(95 \%$ confidence interval $[\mathrm{CI}], 2,43.3), 73.8 \%$ mild $(95 \% \mathrm{CI}, 42.8,82)$ and $14.3 \%$ no $(95 \%$ CI, $2.9,50.7)$ mitral valve stenosis. The detailed mid-term results of echocardiographic follow-up are summarized in Table 2, illustrating a slight but not significant decrease of mean left ventricular end diastolic diameter from $57.5 \mathrm{~mm}(49.0-65.2)$ to 52.1 (47.3-59.2) $(P=.074)$ at latest follow-up. Video 1 provides a summary of important findings of the manuscript. A graphical abstract is illustrated in Figure 4.

\section{DISCUSSION}

Moderate or moderate-to-severe mitral valve regurgitation may present as a concomitant finding in patients requiring a challenging aortic root/valve or left ventricular remodeling procedure. This particular patient cohort represents frequently a multimorbid, high-risk population, as expressed in our series with an elevated EuroSCORE II of $11.4 \%(6.54 \%-14.9 \%)$.

Etiology of mitral valve regurgitation included valvular or ischemic cardiomyopathy leading to a secondary mitral valve insufficiency. Recommendations regarding the treatment of moderate or moderate-to-severe secondary mitral 
TABLE 4. Proportion of patients in each mitral valve stenosis grade over time

\begin{tabular}{|c|c|c|c|c|c|c|}
\hline & $1 \mathbf{y}, \%(95 \%$ CI $)$ & $2 \mathbf{y}, \%(95 \%$ CI $)$ & $3 \mathrm{y}, \%(95 \% \mathrm{CI})$ & 4 y, \% $(95 \%$ CI $)$ & $5 y, \%(95 \%$ CI $)$ & $6 \mathbf{y}, \%(95 \% \mathbf{C I})$ \\
\hline \multicolumn{7}{|l|}{ MS grade } \\
\hline Severe & 0 & 0 & 0 & 0 & 0 & 0 \\
\hline Moderate & $13.8(7.1,24.7)$ & $13.2(5.1,28.9)$ & $12.5(3.3,33.6)$ & $11.9(2,43.3)$ & $11.4(1.1,47.8)$ & $10.8(0.5,54.9)$ \\
\hline Mild & $73.6(54,83.3)$ & $73.7(52.7,82.9)$ & $73.8(49.3,82.7)$ & $73.8(42.2,82)$ & $73.7(30.3,81.3)$ & $73.7(20.8,81)$ \\
\hline None & $12.6(5.2,29.1)$ & $13.1(4.7,32.6)$ & $13.7(3.9,41.6)$ & $14.3(2.9,50.7)$ & $14.9(2.3,58)$ & $15.5(1.8,66.7)$ \\
\hline
\end{tabular}

$C I$, Confidence interval; $M S$, mitral stenosis.

valve regurgitation in patients requiring aortic root/valve or left ventricular remodeling procedure are vaguely defined in the current guidelines for the management of valvular heart disease. $^{1,2}$

Robust randomized controlled trials comparing transatrial mitral valve repair versus replacement for functional mitral valve regurgitation suggest superior outcomes regarding the rate of recurrence of moderate or severe mitral regurgitation for patients undergoing mitral valve replacement. ${ }^{18}$ In contrast, the need to make an extra left atrial incision, the commonly laterally displacement of the mitral annulus due to aortic root/left ventricular dilatation, and frequently small left atrium in these cases make the left atrial route in many patients unfavorable.

McCarthy and associates ${ }^{17}$ compared, in a retrospective study, outcomes in a series of 1316 patients undergoing aortic root replacement who did or did not receive a concomitant mitral repair via left atrial approach in the presence of preoperative moderate mitral regurgitation. In their series, crossclamp time increased by more than 1 hour (crossclamp time concomitant mitral valve repair $=244.8 \pm 42$ minutes vs no concomitant mitral valve

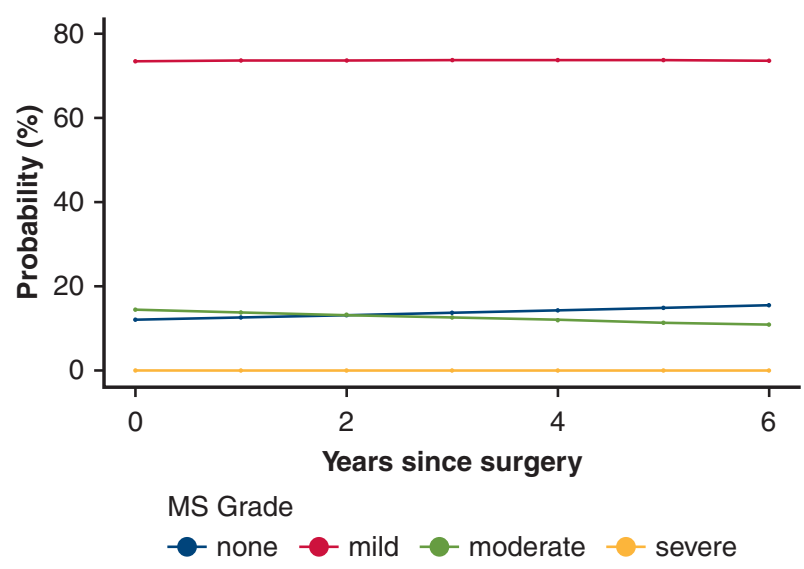

FIGURE 3. The proportion of patients in each mitral valve stenosis grade over time. Four years postoperatively, none of the patients with echocardiographic follow-up had severe mitral valve stenosis (orange curve), whereas $11.9 \%$ of the patients had moderate (green curve, $95 \% \mathrm{CI}, 2,43.3$ ), $73.8 \%$ mild (red curve, $95 \% \mathrm{CI}, 42.8,82$ ), and $14.3 \%$ no (blue curve, $95 \% \mathrm{CI}$, $2.9,50.7)$ mitral valve stenosis. The number of patients who had echocardiographic measurements at each time period is as follows: at 2 years, 19 patients; at 4 years, 10 patients; and finally at 6 years, 5 patients. $C I$, Confidence interval; $M S$, mitral valve stenosis. repair $=179.6 \pm 50.6$ minutes) in patients undergoing additional mitral valve repair.

Although mitral valve regurgitation may decrease after correction of aortic valve pathology or restoration of left ventricular geometry, postoperative persistence of mitral valve regurgitation may occur affecting survival adversely. ${ }^{16,17}$ Thus, a quick and technically simple procedure for the concomitant treatment of moderate or moderateto-severe mitral valve regurgitation avoiding conventional transatrial mitral approach with increased ischemia time is key. Such a procedure may present an effective tool for a concomitant approach of mitral valve regurgitation during aortic root/valve or left ventricular remodeling procedure.

Fucci and associates ${ }^{19}$ introduced the edge-to-edge repair as a simple surgical solution for regurgitant mitral valves back in 1995. This double-orifice mitral valve repair technique has been used in diverse morphologic settings by several groups with satisfactory perioperative and midterm outcomes. ${ }^{21-23}$ Nowadays, the concept is used by percutaneous interventional techniques like MitraClip implantation, especially in high-risk patients, were a reduction in regurgitation but not a perfectly competent valve is the primary goal, showing good results in the setting of the secondary mitral regurgitation. ${ }^{1-3}$ In 2006, we began applying the edge-to-edge repair through a transventricular and transaortic route in high-risk patients requiring left

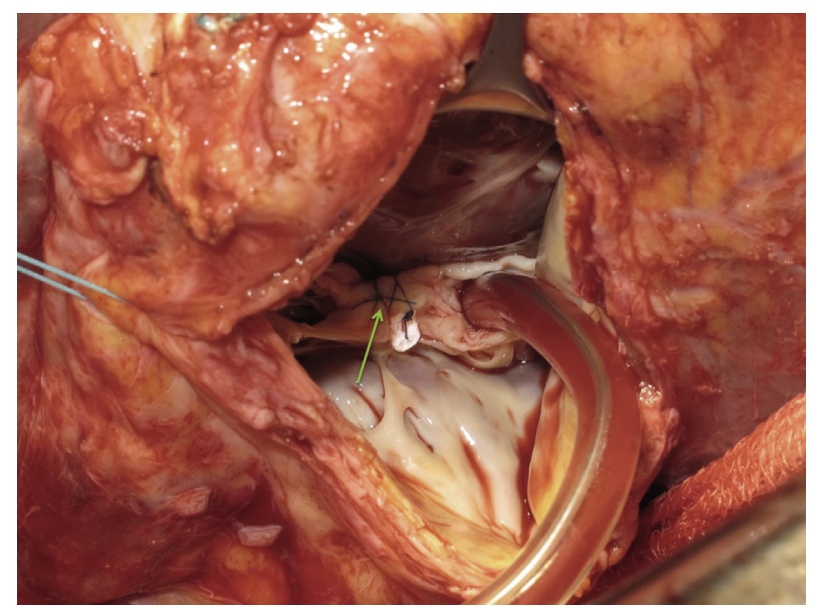

VIDEO 1. Shown is a summary of important findings of the current manuscript. Video available at: https://www.jtcvs.org/article/S2666-2507(22) 00032-3/fulltext. 
Modified Transventricular and Transaortic Mitral Valve Edge-to-Edge Repair

\begin{tabular}{|l|}
\hline \multicolumn{1}{|c|}{ Methods } \\
From 2006 through 2015,49 high-risk patients presented with aortic \\
root or leftventricular aneurysm and concomitant secondary $\mathrm{MR}\left(2^{+}-4^{+}\right)$ \\
-42 underwent transaortic mitral edge-to-edge repair \\
-7 underwent transventricular mitral edge -to-edge repair \\
\hline $\begin{array}{l}\text { Alfieri-Stitch technique was modified by MitraClip type overcorrection } \\
\text { and solid buttressing behind the posterior leaflet. }\end{array}$ \\
\hline
\end{tabular}

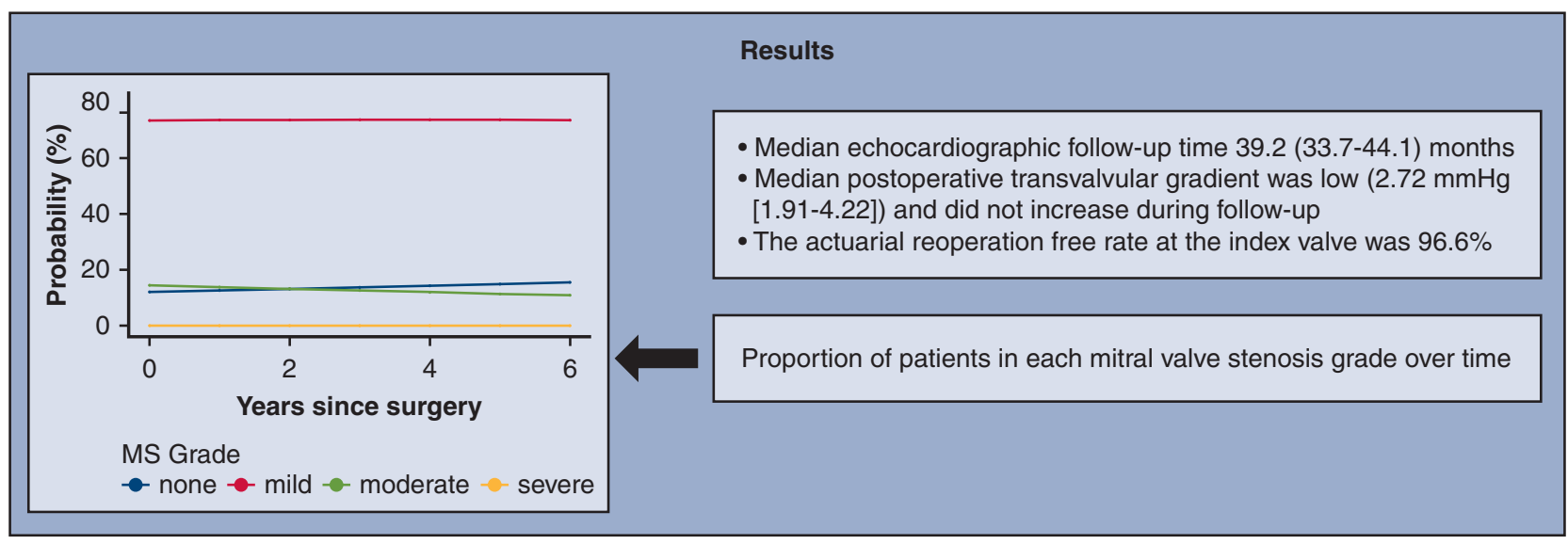

Implications

Transventricular or transaortic Alfieri mitral repair mimicking MitraClip Overcorrection represents a safe technique and can be performed with low risk of creating mitral stenosis at midterm. The technique is straightforward, with reliable identification of the center of the valve leaflets being the limitation

FIGURE 4. Methods: From 2006 through 2015, 49 high-risk patients presented with aortic root or left ventricular aneurysm and concomitant secondary MR $\left(2^{+}-4^{+}\right)$. Forty-two patients underwent transaortic mitral edge-to-edge repair. Seven patients underwent transventricular mitral edge-to-edge repair. The Alfieri stitch technique was modified by MitraClip type overcorrection and solid buttressing behind the posterior leaflet. Results: Median echocardiographic follow-up time 39.2 (33.7-44.1) months. Median postoperative transvalvular gradient was low (2.72 $\mathrm{mm} \mathrm{Hg}$ [1.91-4.22]) and did not increase during followup. The actuarial reoperation free rate at the index valve was $96.6 \%$. Proportion of patients in each mitral valve stenosis grade over time. Implications: Transventricular or transaortic Alfieri mitral repair mimicking MitraClip overcorrection represents a safe technique and can be performed with low risk of creating mitral stenosis at midterm. The technique is straightforward, with reliable identification of the center of the valve leaflets being the limitation. $M R$, Mitral valve regurgitation; $M S$, mitral valve stenosis.

ventricular remodeling or a challenging aortic root/valve surgery. Transventricular or transaortic mitral edge-toedge repair represents a quick and safe alternative once the mitral valve can be approached from the surgical access way of the primary procedure. ${ }^{21-23}$ Frequently dilated left ventricle and aortic root makes visibility of mitral valve easer. In the current study, we present our midterm experience with this procedure.

Only 1 edge-to-edge repair in our series had to be abandoned intraoperatively due to a high transvalvular gradient, leading to a procedural success rate of $98 \%$, which is comparable with other series published in the past. ${ }^{23}$ The most common reason leading to unsatisfactory repair following edge-to-edge repair through the transaortic access is the incorrect identification of P2 segment leading to misplacement of the Alfieri stitch in the posterior mitral leaflet. After a series of anatomical studies and gaining of experience with $\mathrm{P} 2$ center identification in cases of conventional repair of secondary mitral incompetence, it became clear that in the vast majority of the cases the center of the P2 segment is defined by the watershed created from the primary chordae tendineae. Thus, detection of the watershed, as shown in Figure 2 created from primary chordae tendineae originating from the anterolateral and posteromedial pupillary muscles reaching the P2 segment represents a key step for the proper positioning of Alfieri stitch at the posterior mitral leaflet during transaortic edge-to-edge repair. The second most common appearance of the subvalvular apparatus of the posterior mitral leaflet is illustrated in Figure E2, with a fan-shaped flattened 
posterior papillary muscle with broad attachment of P2 chordae without division of direction. In such cases, the maximal height of the P2 segment may indicate the center of the leaflet. However, this is not always precise. An asymmetric prolapse (P1/A1 or P3/A3) is hard to repair by the presented technique, as it is very difficult to identify the corresponding points of anterior and posterior leaflets. In addition, accessibility behind the chordae is limited.

Maisano and associates ${ }^{20}$ reported in a series of $81 \mathrm{pa}-$ tients suboptimal midterm results following edge-to-edge repair technique when annuloplasty ring is not added to the repair. As annuloplasty is neither through the transaortic nor through the transventricular access feasible, we modified the Alfieri stitch technique by passing a pledgetreinforced stitch near the annulus of the P2 segment, whereas the second arm is placed close to the free edge. The distance in the anterior mitral leaflet (A2 segment) was chosen dependent on the height of the leaflet. In this manner, effectivity of the technique can be improved by mimicking a MitraClip style overcorrection through solid buttressing behind the posterior leaflet that may lead to a reduction in the anteroposterior diameter of the mitral annulus. It is important to firmly buttress the P2 stitch, as the leaflet may be friable. In this scenario, a piece of Teflon felt can be added to the more rigid pledget.

Based on our midterm echocardiographic findings, transventricular and transaortic mitral valve edge-to-edge repair can be applied with a low risk of creating mitral valve stenosis. Echocardiographic follow-up data beyond 1.5 years postoperatively are scarcely described in the literature following edge-to-edge repair via transaortic and transventricular route. Bhudia and associates ${ }^{23}$ reported in a series of 224 patients about a low, nonprogressive mean transvalvular gradient of $3.7 \mathrm{~mm} \mathrm{Hg}$ following Alfieri mitral valve repair in early follow-up. However, in their series, only $20 \%$ of the mitral valves were accessed transventricularly or transaortically. Choudhary and colleagues ${ }^{21}$ reported in a small series of 16 patients an incidence of moderate mitral valve stenosis of $6.25 \%$ at a median echocardiographic follow-up time of 20 months. In our series, longitudinal analysis of serial echocardiographic data revealed an absence of severe mitral valve stenosis and $11.9 \%$ incidence of moderate mitral valve stenosis 4 years postoperatively.

Several publications have reported improvements in functional status following edge-to-edge repair. ${ }^{25,26} \mathrm{Ac}$ cording to our findings, it is difficult to draw a similar conclusion from our series since the disease burden of our patient cohort was not primarily defined by the mitral valve regurgitation. However, it is notable to underline the improvement in the NYHA functional status in our patient cohort at mid-term follow-up, as illustrated in Figure E5, although $9 \%$ and $12 \%$ of patient cohort are still presented with moderate mitral valve regurgitation or stenosis respectively at midterm follow-up.
Oc and coworkers ${ }^{27}$ reported a 5-year freedom from reoperation rate of $94 \%$, in a series of 41 patients following edge-to-edge repair. Similar results have been published in a larger series from Alfieri and colleagues ${ }^{28}$ involving 260 patients undergoing edge-to-edge repair, with freedom from reoperation at 5 years being $90 \%$. We can corroborate these findings, as only 1 patient required reoperation on the index valve at 1 year postoperatively, with the actuarial reoperation-free rate in our series being $96.6 \%$. In our hands, posterior mitral leaflets may be very flaccid and thus stitches need to be reinforced. Usually, Teflon pledgets were used in our series. In the single reoperated case, an Alfieri stitch was buttressed with autologous pericardium, as the primary indication for surgery was active aortic valve endocarditis. This stitch ruptured early after surgery, leading to reoperation of the index valve 1 year postoperatively.

\section{Study Limitations}

The study is limited by its retrospective, single-center descriptive nature. No conclusions regarding the comparative efficacy of the described technique versus others (Alfieri stitch, annuloplasty repair, replacement) can be inferred. A further weakness of the manuscript represents the heterogeneous nature of primary procedures. Robust randomized controlled trials comparing mitral valve repair versus replacement for functional mitral valve regurgitation suggest superior outcomes for patients undergoing mitral valve replacement. ${ }^{18}$ This conclusion gives rise to the question of whether a shorter bypass time with the modified Alfieri stitch outweighs the proven benefit in patients undergoing mitral valve replacement for functional mitral valve regurgitation. Regardless, this study cannot comment on this comparison, which represents another limitation of the current manuscript. Frequently dilated left ventricle and aortic root makes visibility of mitral valve easer. However, in patients with an absence of aortic root dilatation, the surgical view of the mitral valve through the aortic valve may be inferior to the view from the left atrium.

\section{CONCLUSIONS}

Transventricular or transaortic Alfieri mitral repair represents a quick and safe technique in the setting of high-risk patients undergoing left ventricular remodeling or aortic root/valve surgery and can be performed with low risk of creating mitral stenosis at midterm. The technique is straightforward, with reliable identification of the center of the valve leaflets being the limitation. Effectivity of the technique may be improved by MitraClip type overcorrection and solid buttressing behind the posterior leaflet.

\section{Conflict of Interest Statement}

The authors reported no conflicts of interest.

The Journal policy requires editors and reviewers to disclose conflicts of interest and to decline handling or 
reviewing manuscripts for which they may have a conflict of interest. The editors and reviewers of this article have no conflicts of interest.

\section{References}

1. Nishimura RA, Otto CM, Bonow RO, Carabello BA, Erwin JP III, Fleisher LA, et al. 2017 AHA/ACC focused update of the 2014 AHA/ACC guideline for the management of patients with valvular heart disease: a report of the American College of Cardiology/American Heart Association task force on clinical practice guidelines. Circulation. 2017;135:1159-95.

2. Baumgartner H, Falk V, Bax JJ, De Bonis M, Hamm C, Holm PJ, et al; ESC Scientific Document Group. 2017 ESC/EACTS Guidelines for the management of valvular heart disease. Eur Heart J. 2017;38:2739-91.

3. Nishimura RA, Otto CM, Bonow RO, Carabello BA, Erwin JP III, Guyton RA, et al; American College of Cardiology, American College of Cardiology/American Heart Association, American Heart Association. 2014 AHA/ACC guideline for the management of patients with valvular heart disease: a report of the American College of Cardiology/American Heart Association task force on practice guidelines. J Thorac Cardiovasc Surg. 2014;148:1-132.

4. Jouan J, Berrebi A, Chauvaud S, Menasché P, Carpentier A, Fabiani JN. Mitral valve reconstruction in Barlow disease: long-term echographic results and implications for surgical management. J Thorac Cardiovasc Surg. 2012;143:17-20.

5. Iung B, Rousseau-Paziaud J, Cormier B, Garbarz E, Fondard O, Brochet E, et al. Contemporary results of mitral valve repair for infective endocarditis. J Am Coll Cardiol. 2004:43:386-92.

6. Stellin G, Padalino MA, Vida VL, Boccuzzo G, Orrù E, Biffanti R, et al. Surgical repair of congenital mitral valve malformations in infancy and childhood: a single-center 36-year experience. Thorac Cardiovasc Surg. 2010;140:1238-44.

7. Morimoto H, Tsuchiya K, Nakajima M, Akashi O, Kato K. Mitral valve repair for extended commissural prolapse involving complex prolapse. Asian Cardiovasc Thorac Ann. 2007;15:210-3.

8. Castillo JG, Anyanwu AC, Fuster V, Adams DH. A near 100\% repair rate for mitral valve prolapse is achievable in a reference center: implications for future guidelines. J Thorac Cardiovasc Surg. 2012;144:308-12.

9. Feindel CM, Tufail Z, David TE, Ivanov J, Armstrong S. Mitral valve surgery in patients with extensive calcification of the mitral annulus. J Thorac Cardiovasc Surg. 2003; 126:777-82

10. Grossi EA, Galloway AC, Steinberg BM, LeBoutillier M III, Delianides J, Baumann FG, et al. Severe calcification does not affect long-term outcome of mitral valve repair. Ann Thorac Surg. 1994:58:685-7.

11. Barreiro CJ, Patel ND, Fitton TP, Williams JA, Bonde PN, Chan V, et al. Aortic valve replacement and concomitant mitral valve regurgitation in the elderly. Circulation. 2005;112:443-7.

12. Regeer MV, Al Amri I, Versteegh MI, Bax JJ, Marsan NA, Delgado VJ. Mitral valve geometry changes in patients with aortic regurgitation. Am Soc Echocardiogr. 2015;28:455-62.

13. Petrus AHJ, Klein P, Tops LF, Dekkers OM, Hoogervorst LA, Couperus LE, et al. 10-year outcomes after left ventricular reconstruction: rethinking the impact of mitral regurgitation. Ann Thorac Surg. 2019;108:81-8.
14. Song Y, Hu S, Sun H, Song Y, Wang L, Wang W, et al. Results of left ventricular reconstruction with and without mitral valve surgery. Ann Thorac Surg. 2020; 109:753-61

15. Rankin JS, Hammill BG, Ferguson TB Jr, Glower DD, O’Brien SM, DeLong ER, et al. Determinants of operative mortality in valvular heart surgery. J Thorac Cardiovasc Surg. 2006;131:547-57.

16. Pai RG, Varadarajan P. Prognostic implications of mitral regurgitation in patients with severe aortic regurgitation. Circulation. 2010;122:43-7.

17. McCarthy FH, Desai ND, Fox Z, George J, Moeller P, Vallabhajosyula P, et al Moderate mitral regurgitation in aortic root replacement surgery: comparing mitral repair with no mitral repair. J Thorac Cardiovasc Surg. 2014;147:938-41.

18. Goldstein D, Moskowitz AJ, Gelijns AC, Ailawadi G, Parides MK, Perrault LP, et al; CTSN. Two-year outcomes of surgical treatment of severe ischemic mitral regurgitation. N Engl J Med. 2016;374:344-53.

19. Fucci C, Sandrelli L, Pardini A, Torracca L, Ferrari M, Alfieri O. Improved results with mitral valve repair using new surgical techniques. Eur J Cardiothorac Surg. 1995;9:621-6.

20. Maisano F, Caldarola A, Blasio A, De Bonis M, La Canna G, Alfieri O. Midterm results of edge-to-edge mitral valve repair without annuloplasty. J Thorac Cardiovasc Surg. 2003;126:1987-97.

21. Choudhary SK, Abraham A, Bhoje A, Gharde P, Sahu M, Talwar S, et al. Transaortic edge-to-edge mitral valve repair for moderate secondary/functional mitra regurgitation in patients undergoing aortic root/valve intervention. J Thorac Cardiovasc Surg. 2017;154:1624-9.

22. Brinster DR, Unic D, D'Ambra MN, Nathan N, Cohn LH. Midterm results of the edge-to-edge technique for complex mitral valve repair. Ann Thorac Surg. 2006 81:1612-7.

23. Bhudia SK, McCarthy PM, Smedira NG, Lam BK, Rajeswaran J, Blackstone EH Edge-to-edge (Alfieri) mitral repair: results in diverse clinical settings. Ann Thorac Surg. 2004;77:1598-606.

24. Nashef SA, Roques F, Michel P, Gauducheau E, Lemeshow S, Salamon R. Euro pean system for cardiac operative risk evaluation (EuroSCORE). Eur J Cardiothorac Surg. 1999;16:9-13.

25. De Bonis M, Lapenna E, La Canna G, Ficarra E, Pagliaro M, Torracca L, et al Mitral valve repair for functional mitral regurgitation in end- stage dilated cardiomyopathy: role of the "edge-to-edge" technique. Circulation. 2005;112:402-8.

26. Raman J, Shah P, Seevanayagam S, Cheung J, Buxton B. Mitral regurgitation comparison between edge-to-edge repair and valve replacement. Asian Cardiovasc Thorac Ann. 2003;11:131-4.

27. Oc M, Doukas G, Alexiou C, Oc B, Hadjinikolaou L, Sosnowski AW, et al. Edgeto-edge repair with mitral annuloplasty for Barlow's disease. Ann Thorac Surg. 2005;80:1315-8.

28. Alfieri O, Maisano F, De Bonis M, Stefano PL, Torracca L, Oppizzi M, et al. The double-orifice technique in mitral valve repair: a simple solution for complex problems. J Thorac Cardiovasc Surg. 2001;122:674-81.

Key Words: transaortic mitral valve repair, transventricular mitral valve repair, edge-to-edge repair, functional mitral valve regurgitation 


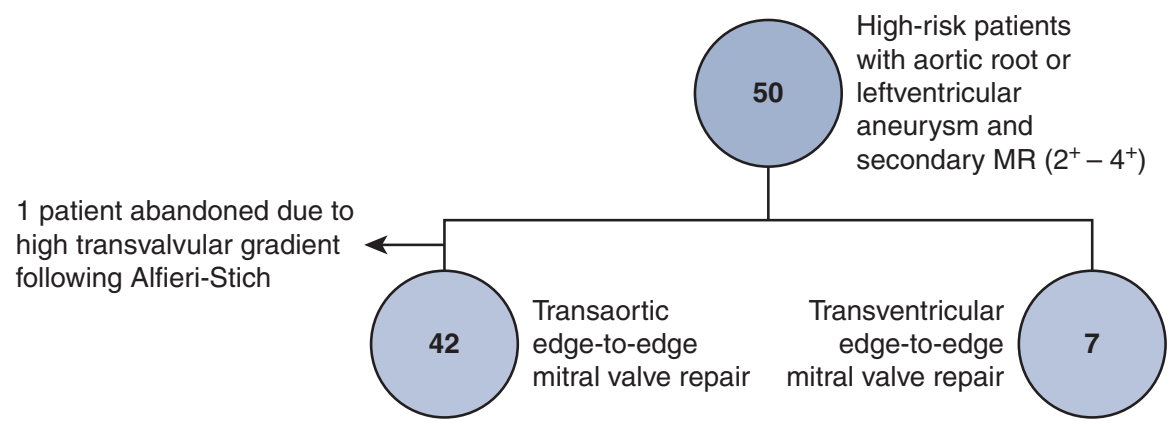

FIGURE E1. A consort diagram of our study. High-risk patients requiring aortic root surgery or left ventricular remodeling due to aortic root or left ventricular aneurysm in the presence of a secondary mitral valve regurgitation were eligible for transaortic or transventricular edge-to-edge repair. From December 2006 through April 2015, 7 patients (14\%) underwent concomitant modified edge-to-edge mitral valve repair through a transventricular and 43 patients $(86 \%)$ through a transaortic route. In the transaortic group, 1 procedure $(2 \%)$ had to be abandoned intraoperatively due to a high transvalvular gradient. This patient was successfully treated by takedown of the edge-to-edge approximation and performance of mitral valve reconstruction using a standard annuloplasty-ring during a second pump run. $M R$, Mitral valve regurgitation.

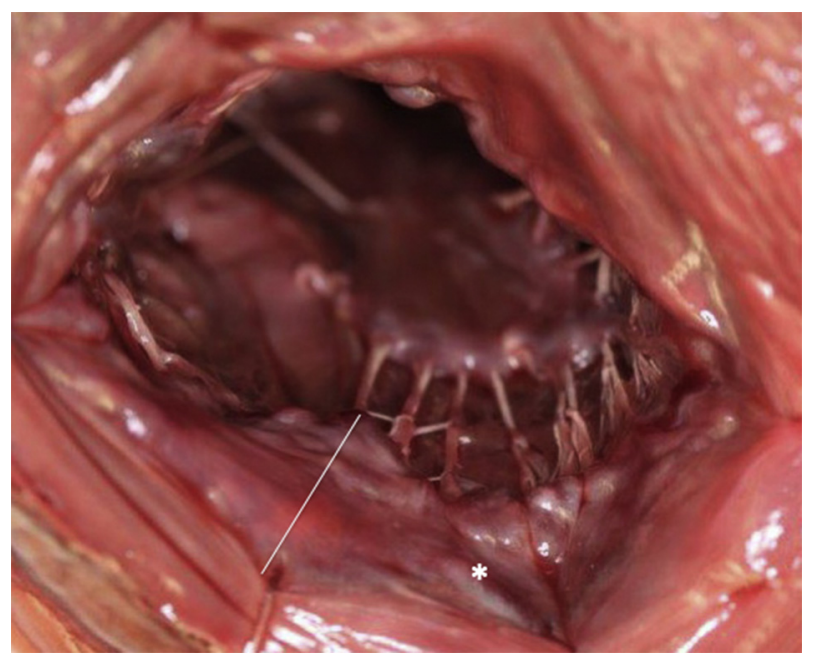

FIGURE E2. Surgical view after transseptal approach of mitral valve. Posterior mitral leaflet is illustrated at the bottom (single white asterisk). The subvalvular apparatus of the posterior mitral leaflet is illustrated with a fan-shaped, flattened posterior papillary muscle with broad attachment of P2 chordae without division of direction. This represents a rare but possible anatomical arrangement of subvalvular apparatus. In such cases the detection of $\mathrm{P} 2$ segment via transaortic or transventricular route is challenging once a watershed of chordae tendinae originating from anterolateral and posteromedial papillary muscle indicating P2 segment is absent. The maximal height (white line) of the $\mathrm{P} 2$ segment may indicate the center of the leaflet. 


\section{Edge-to-edge Repair}

Transaortic route

$\mathrm{N}=42(86 \%)$

\begin{tabular}{|lc|}
\hline Bentall's Procedure & $\mathrm{N}=6$ \\
T.David's Procedure & $\mathrm{N}=6$ \\
Bentall + Hemiarch Repl. $\mathrm{N}=4$ \\
Bentall + CABG & $\mathrm{N}=10$ \\
Bentall + TVR & $\mathrm{N}=6$ \\
\hline
\end{tabular}

Transventricular route $\mathrm{N}=7(14 \%)$

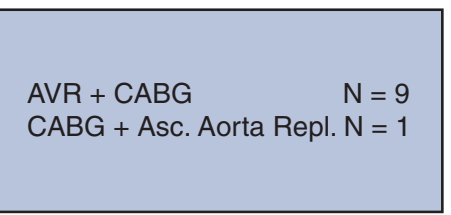

CABG + Dor-Plasty $\mathrm{N}=7$

FIGURE E3. Overview of surgical procedures. Forty-two patients ( $86 \%)$ underwent transaortic mitral valve edge-to-edge repair. Among them, 32 patients $(65 \%)$ underwent aortic root replacement with reimplantation $(\mathrm{N}=6)$ or replacement $(\mathrm{N}=26)$ of the aortic root. Nine patients $(18 \%)$ required aortic valve replacement $(A V R)$ with concomitant coronary artery bypass grafting $(C A B G)$. In one case $(2 \%)$ transaortic edge-to-edge repair was performed in a second pump run following replacement of the ascending aorta and CABG due to a progression of mitral valve regurgitation from moderate to severe. Seven patients $(\mathrm{N}=14 \%$ ) required CABG and left ventricular remodeling (Dor-Plasty). Thus, in those patients, mitral valve edge-to-edge repair was performed through a transventricular approach. TVR, Tricuspid valve repair.

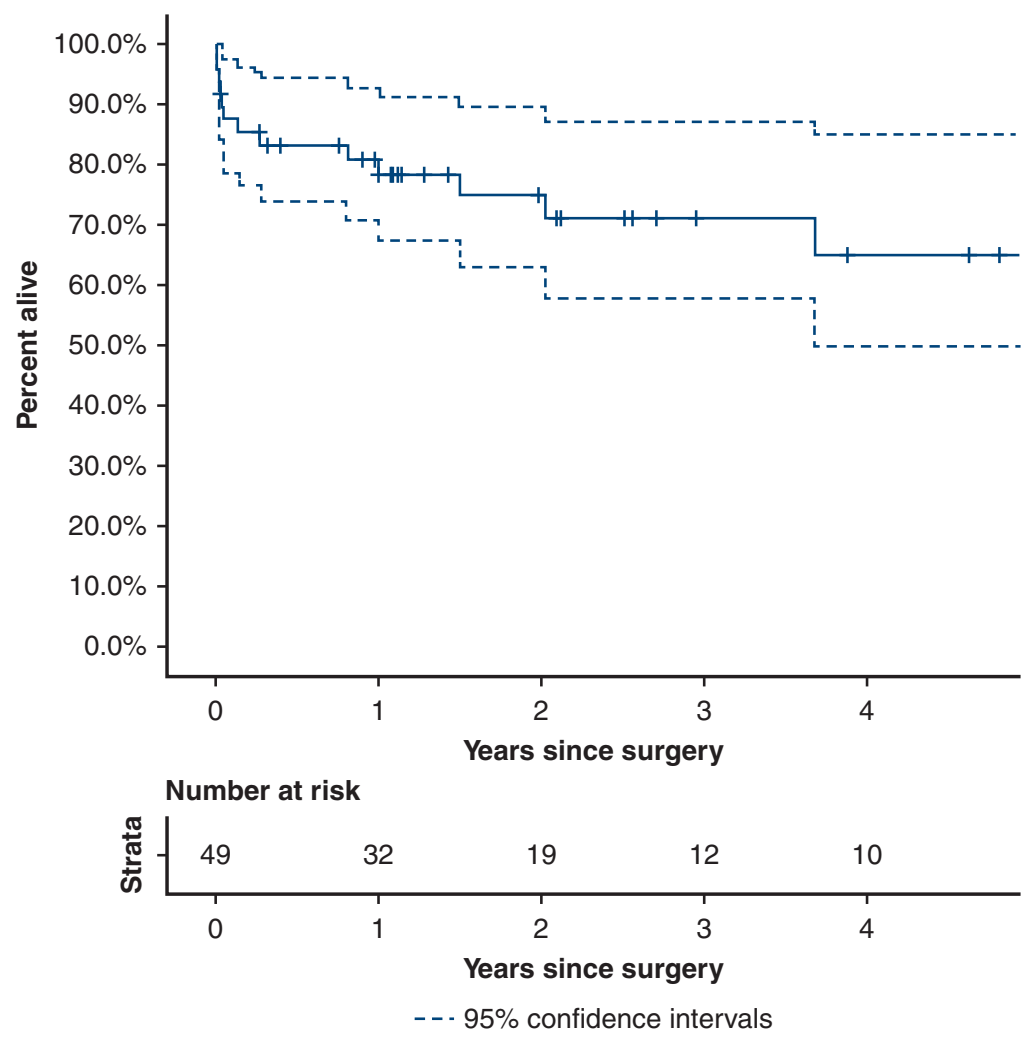

FIGURE E4. Based on Kaplan-Meier analysis, overall survival was $66.5 \pm 2 \%$ at 4 years. 


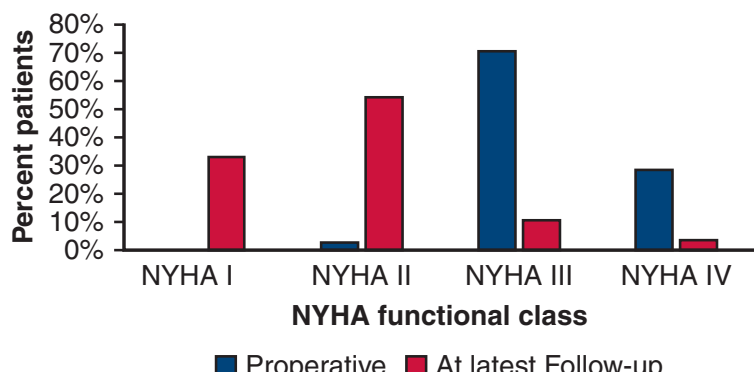

FIGURE E5. The distribution of New York Heart Association (NYHA) classification before cardiac surgery and at latest follow-up. Preoperatively, the vast majority of the patients were in NYHA functional class III or IV $(\mathrm{N}=48 ; 98 \%)$. At latest follow-up 10 of the 30 survivors $(33 \%)$ were in NYHA functional class I, $16(54 \%)$ in class II, $3(10 \%)$ in class III, and 1 patient $(3 \%)$ in class IV. 
TABLE E1. Echocardiographic outcomes between transaortic and transventricular approach at latest follow-up

\begin{tabular}{|c|c|c|c|c|c|}
\hline \multirow{3}{*}{$\begin{array}{l}\text { Variables at latest follow-up } \\
\text { Max MVP, } \mathrm{mm} \mathrm{Hg}\end{array}$} & \multicolumn{2}{|r|}{ Transaortic } & \multicolumn{2}{|c|}{ Transventricular } & \multirow[b]{2}{*}{$P$ value } \\
\hline & \multicolumn{4}{|c|}{ Median (interquartile range) } & \\
\hline & \multicolumn{2}{|r|}{$8.12(6.17-13.6)$} & \multicolumn{2}{|c|}{$7.61(6.22-13.7)$} & .146 \\
\hline Mean MVP, mm Hg & \multicolumn{2}{|r|}{$3.21(2.34-4.72)$} & \multicolumn{2}{|c|}{$2.92(2.44-4.22)$} & .543 \\
\hline Mean MVA, $\mathrm{cm}^{2}$ & \multicolumn{2}{|r|}{$2.24(2.11-2.64)$} & \multicolumn{2}{|c|}{$2.021(2.011-2.72)$} & .618 \\
\hline Left atrial diameter, $\mathrm{mm}$ & \multicolumn{2}{|r|}{$45.2(39.3-51.6)$} & \multicolumn{2}{|c|}{$44.7(40.8-50.1)$} & .754 \\
\hline \multicolumn{6}{|l|}{ Mitral valve regurgitation grade } \\
\hline None & 3 & 0 & 0 & 0 & \\
\hline $1+$ & 14 & 0 & 4 & $56.2 \%$ & \\
\hline $2+$ & 8 & $52 \%$ & 0 & 0 & \\
\hline $3+$ & 2 & $45 \%$ & 1 & $9.3 \%$ & \\
\hline $4+$ & 0 & 0 & 0 & 0 & \\
\hline
\end{tabular}

$M V P$, Mitral valve pressure gradient; $M V A$, mitral valve area. 